\title{
Laboratory Studies in Support of the Exploration of Ocean Worlds and NASA Missions
}

A white paper for the 2023-2032 Planetary Science and Astrobiology Decadal Survey

Abstract: There are no perfect ocean-worlds analog environments on Earth, and so laboratory experiments, spectral databases, and computational work are needed to simulate broad and truly abiotic planetary conditions that can provide important constraints for technological development and plans for exploration by telescopes, orbiting spacecraft, and landers.

Lead Author: Reggie L. Hudson, NASA Goddard Space Flight Center, (301) 286- 6961, reggie.hudson@nasa.gov

Co-authors: Bethany Theiling ${ }^{1}$, Dina Bower ${ }^{1,2}$, Heather Graham ${ }^{1,3}$, Melissa Trainer ${ }^{1}$, Conor Nixon ${ }^{1}$, Stefanie Milam 1

Endorsers: Veronica Allen ${ }^{1,4}$, Gordon Bjoraker ${ }^{1}$, Jason Dworkin ${ }^{1}$, Jennifer Eigenbrode ${ }^{1}$, Perry Gerakines $^{1}$, Christopher Materese ${ }^{1}$, Jennifer Stern ${ }^{1}$, Geronimo Villanueva ${ }^{1}$

Affiliations: ${ }^{1}$ NASA Goddard Space Flight Center, ${ }^{2}$ University of Maryland College Park, ${ }^{3}$ Catholic University of America, ${ }^{4}$ Universities Space Research Association
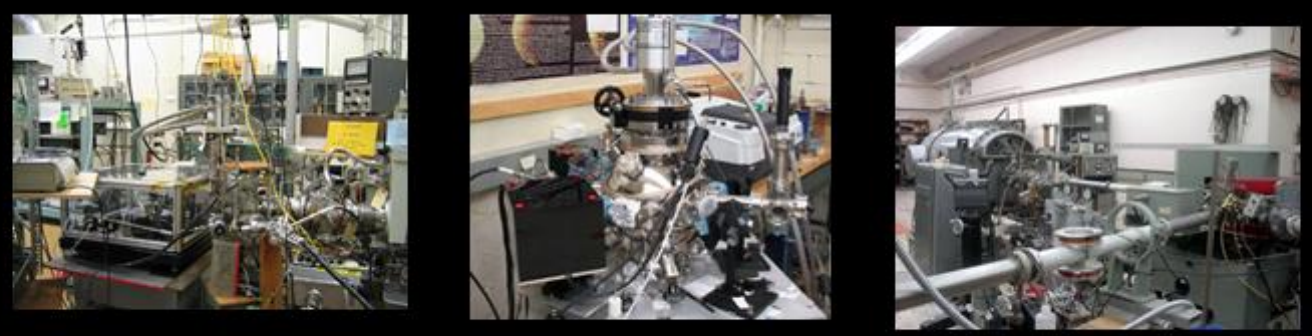

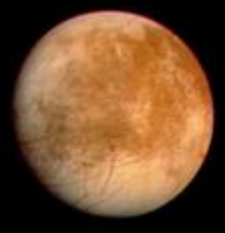

Europa

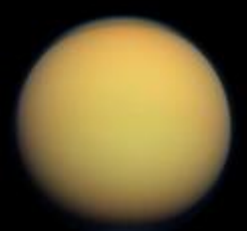

Titan

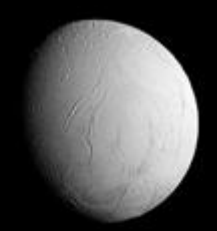

Enceladus

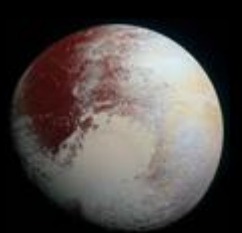

Pluto

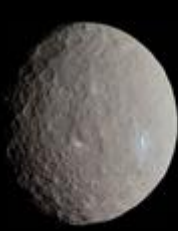

Ceres 


\section{Laboratory Studies in Support of the Exploration of Ocean Worlds and NASA Missions}

Lead Author: Reggie Hudson Co-authors: Bethany Theiling, Dina Bower, Heather Graham, Melissa Trainer, Conor Nixon, Stefanie Milam Endorsers: Veronica Allen, Gordon Bjoraker, Jason Dworkin, Jennifer Eigenbrode, Perry Gerakines, Christopher Materese, Jennifer Stern, Geronimo Villanueva

\section{Executive Summary}

"Vision and Voyages for Planetary Science in the Decade 2013-2022", the mostrecent planetary science decadal survey (hereafter $\mathrm{V} \& \mathrm{~V}$ ) explicitly notes the importance of fundamental scientific research as "the foundation for interpreting data collected by spacecraft, as well as the guidance and context for identifying new scientifically compelling missions" (p. 21), and that laboratory work is "crucially important to NASA's long-term science goals" (p. 21). Highlighted in V\&V are the importance of laboratory simulations, measurements of fundamental properties, and spectroscopic databases ( $p$. 29). V\&V describes research as having a "central role" and as "vital to planetary exploration" (p. 300), sentiments that have motivated fundamental and analog research over the past decade. Here we describe several key laboratory methods, instruments, and chemical systems that are important for the next decade of ocean-worlds exploration, and we make recommendations for lab studies and development of spectral libraries to support planetary missions.

\section{Introduction}

Laboratory challenges for the ocean-worlds exploration are as varied as the worlds themselves. Planetary targets of interest cover such diversity as high-radiation environments (Europa), the presence of plumes (Enceladus, Triton), atmospheric pressure greater than Earth's (Titan), and worlds dominated by solid nitrogen (Pluto), rocky surfaces (Ceres), or ice (Europa, Enceladus, Ganymede, Callisto).

This diversity, combined with the paucity of data from these targets, motivates lab research to develop predictive conceptual and computational (e.g., geophysical, geochemical) models to aid in analysis and interpretation of future mission data. Therefore, a key issue for laboratory scientists interested in ocean worlds is in constraining the relevant

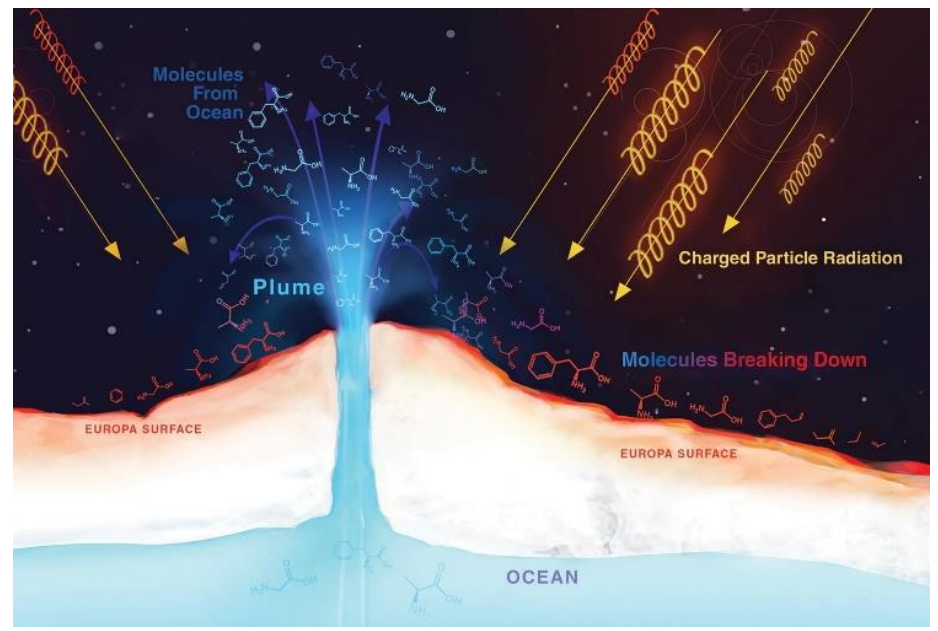

Figure 1. Activities on, above, and below an ocean world's surface contribute to a dynamic chemical, geological, and potentially biological system. Laboratory research enables an understanding of a planetary system under varying conditions before mission data collection. Image: NASA/JPL-Caltech 
chemical and physical conditions for each planetary target, including the nature of surface and sub-surface activity, chemical cycling, geological interaction, and evolution of the system (e.g., Europa, Figure 1). Since there are no perfect analog environments of ocean worlds on Earth, laboratory studies (1) are required to simulate broad and truly abiotic planetary conditions and (2) can provide important constraints for technological development and plans for exploration by telescopes, orbiting spacecraft, and landers.

Among the more important issues facing laboratory scientists are the types and abundances of chemical species expected in ocean-world environments. For example, it is not clear what composition or concentration of salts characterize Europa's ocean, plumes, or ice shell, which could have profound implications for analysis and interpretation of detected volatiles by the Europa Clipper mission or in situ analyses by the proposed Europa Lander mission. Other ocean-worlds targets host both organic and inorganic species, such as with the well-known case of Titan (e.g., $\mathrm{HCN}$ and $\mathrm{N}_{2}$ vs. $\mathrm{CH}_{4}$ and $\mathrm{CH}_{3} \mathrm{CN}$ ). The fragility of many molecules to temperature and both particle and photonic radiation has been studied by a few research groups (e.g., Hudson et al., 2001; Barnett et al., 2012), but not always with ocean worlds in mind. For Titan, it is important to understand atmospheric photochemical and prebiotic reactions (Trainer et al., 2012) that can be important for the origin of life, and therefore powerful for future data analysis and interpretation for the Dragonfly mission. The extent to which organics in ocean materials are preserved upon escape via plumes is not completely known, but recent experiments have shown that some molecules of interest survive intact (Klenner et al., 2020). In combined organic and inorganic systems, questions related to the mixing of solids, the amorphous or crystalline forms of solids, co-crystals, and the trapping of gases all are relevant. For the latter, the possibility of making inclusion compounds, such as clathrates, on ocean worlds is sometimes proposed as a way to trap volatile materials, but little is known about such solids other than how to make and characterize them. Organic and inorganic icy mixtures containing salts are of interest and relevance for systems such as Europa, Enceladus, and Ceres, but much less studied compared to covalent compounds trapped in icy environments.

This white paper describes powerful examples of ground-based and flightrelevant laboratory technology and techniques as well as general considerations for chemistry and possible biosignatures in ocean-worlds analog environments.

\section{Laboratory Methods}

Several "work horse" techniques have emerged in the past few decades and will be of considerable value for ocean-worlds studies. These include, but are not limited to, radio and microwave spectroscopy, infrared spectroscopy, Raman spectroscopy, mass spectrometry, and large-molecule analysis, and each is described below.

\subsection{Radio- and microwave spectroscopy}

It is doubtful that many techniques will be able to surpass radio- and microwave spectroscopy for remote identification of atmospheric or plume molecular components (e.g., Thelen et al, 2019). Relevant studies require laboratory spectra as reference data 
for comparison to telescopic or spacecraft results. Therefore, databases of radio- and microwave spectra, collected over a range of relevant temperatures, are necessary for future observational analyses. Also needed is support for computational studies (e.g., ab initio calculations) to aid in spectral assignments.

\subsection{Infrared spectroscopy}

Infrared (IR) spectroscopy is the premier method for remote detection of many solid molecular components on ocean-world surfaces. Examples include $\mathrm{H}_{2} \mathrm{O}_{2}$ on Europa, $\mathrm{N}_{2}$ on Pluto, and $\mathrm{CO}_{2}$ and $\mathrm{H}_{2} \mathrm{O}$ on multiple bodies in the outer solar system (e.g., Carlson et al. 2009). Explorations of surface and sub-surface chemistry in cold ocean-worlds environments require IR lab studies with icy solids to explain telescopic and spacecraft results and make predictions to guide new work. For example, Moore and Hudson (2000) and Loeffler and Hudson (2013) studied radiation-induced and thermally induced changes, respectively, of $\mathrm{H}_{2} \mathrm{O}$ and $\mathrm{H}_{2} \mathrm{O}_{2}$, at Europa temperatures to better understand molecular distributions on the Galilean satellites. Aside from simply identifying and predicting ions and molecules on an ocean world, IR methods often are called on to provide abundance data. This is a difficult challenge due to the paucity of lab data on IR band intensities. There is a pressing need for IR band strengths and optical constants for ions and molecules that can be studied to guide our understanding of ocean-worlds chemistry and predict the unknown. In short, reference spectra and tables of not only IR wavelengths of chemical species, but also IR intensities, are needed over a range of temperatures and under variable radiation environments.

\subsection{Raman spectroscopy}

Raman spectra are of limited value for remote sensing, but can be invaluable for in situ measurements. Raman spectroscopy complements IR spectroscopy by providing insight into the composition and physical state of solids and liquids. With a long geoscience heritage, many laboratory Raman measurements have focused on mineral samples, but in recent years Raman has become a go-to method for probing a wide variety of materials including different types of organics and ices. However, there are few relevant laboratory studies showing how Raman might reveal the influence of ionizing radiation on icy mixtures. More must be done to prepare for in situ measurements of ocean-world surfaces that experience UV, magnetospheric, or cosmic-ray exposure, with the accompanying chemical alterations. Raman studies share with IR methods the challenge of making spectral identifications in mixed samples that are unique and quantitative, as opposed to simply suggestive. More work is needed to simulate a broader range of ocean-worlds scenarios using a combination of high-resolution and portable Raman instrumentation. In particular, new studies are needed at temperatures representative of worlds from at least $\sim 100 \mathrm{~K}$ (Europa, surface) to $\sim 40 \mathrm{~K}$ (Pluto, surface).

\subsection{Mass spectrometry}

Mass spectrometers have been used on missions ranging from the solar photosphere to the Moon, Venus, Mars, Jupiter, and Saturn (Titan). Mass spectrometry has been used to determine the composition of soils, rocks, and atmospheres, and has 
advanced our understanding of the origin, differentiation and evolution of the solar system, as well as geologic and atmospheric processes in unique and extreme environments. Mass spectrometry can also be used in combination with other analytical techniques, such as Raman spectroscopy, to provide comprehensive and complementary analyses of complex samples. Aqueous environments such as ocean worlds offer additional challenges for mission-based mass spectrometry, from sample collection, to sample introduction and pre-processing, to analysis and data transmission. Laboratory analog research offers the best opportunity to simulate unique aqueous systems in conditions extreme in radiation, temperature, pressure, and chemistry. The versatility of mass spectrometry across varying sample types provides a framework to study chemical reactions for many possible planetary conditions, and such studies can significantly reduce ambiguities and analysis and interpretation times for transmitted data. For example, lab studies of radiation effects on Europa surface ices can establish a framework for interpreting the source of reaction products identified during a flyby (using IR spectroscopy) or with a lander (using mass spectrometry). Similarly, lab simulations of impact environments on Titan, involving relevant organics and conditions, can prepare for the interpretation of compositional measurements to be made by the Dragonfly mission.

\subsection{Large-molecule analysis}

The laboratory methods mentioned so far are, in themselves, of limited value for detection, identification, and characterization of some of the larger molecules and polymers expected in biological systems. In particular, the sequencing of amino acids, nucleobases, and other monomers are of substantial interest for astrobiology and planetary science. Further, sequencing power can be used for generalized mapping of surface complexity enhancing additional functionality (Johnson et al., 2018). With the advent of portable instrumentation, such as MinION technology, necessary tests have already been conducted on the International Space Station (Castro-Wallace et al., 2017), but more lab research into the response of such technology in simulated oceanworlds analog chemical and physical environments is needed. This will include the exposure of such instruments to relevant variations in temperature and radiation and chemical environments, with samples taken from a variety of ice and mineral sources.

It must be emphasized that with all of these methods, scientific understanding and predictability will come only by examining both simple and complex chemical systems under tightly constrained, planetary-relevant conditions.

\section{Chemical Processes}

The preceding recognizes that chemical and physical changes are and have been active on ocean worlds, and by extension organic chemistry could produce a range of prebiotic (required for the origin of life) and possibly biotic organic molecules. The question of "What can form and how fast?" is important, but rarely addressed as often as necessary. For example, reaction kinetics in the condensed phase presents challenges not readily solved by any known form of computation, unlike gas-phase 
studies. Rate constants for thermal, photochemical, and radiolytic changes are known for few solid-phase processes. One problem that has been addressed by several groups in recent years involves molecular destruction, alteration, or preservation of molecules that could be biomarkers or evidence of terrestrial contamination (e.g., Gerakines et al., 2012). This has been studied extensively in terrestrial environments and for the mineral environments on Mars, but much less data is available for the icy surfaces on ocean worlds. Laboratory research provides the best opportunity to create and test reaction pathways and mechanisms, used in computational models, by allowing for careful testing and discrimination of specific relevant variables, such as alteration of reactants under relevant environments.

The variety of conditions on ocean worlds, both surface or sub-surface, impacts all studies of chemical change involving organic molecules and leads to numerous complications not always appreciated. Consider, for example, the simplest amino acid, glycine, shown in Figure 2, illustrating the structure of gasphase glycine molecules that might be found in a plume or an atmosphere of an ocean world. This is the molecular form often found in textbooks, particularly those for non-specialists, but can be misleading in its simplicity. Due to the unique pressure and<smiles>NCC(=O)O</smiles>

Figure 2. Gas-phase structure of glycine, the simplest amino acid. temperature conditions on ocean worlds, it is necessary to examine the three liquidphase structures of the molecule shown in Figure $\mathbf{3}$ in order to understand the condensed-phase chemistry.
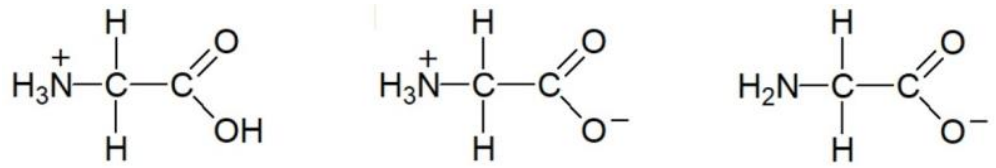

Figure 3. Liquid-phase structures of glycine that would be prevalent under different planetary conditions.

The leftmost structure in Figure 3 is the one expected on an acidic surface, such as that of Europa, and the rightmost is the form that is more relevant where ammonia, a base, is expected, such as Titan. The central structure will be present in environments that are neither acidic nor alkaline, but neutral instead. Looking for the wrong molecular form of, for example, glycine in the wrong environment could lead to a missed discovery, but the identification of any of these forms of glycine would immediately reveal environmental information.

This shows the necessity of examining specific molecular types, such as isomers or tautomers, to determine which are most likely to form and persist in ocean-world environments. One example is the well-known highly stable aromatic compounds, which includes nucleobases. In short, the added information inherent in molecular structure necessitates that flight-based instrumentation and techniques will need the ability to discriminate between variable molecular structures. Lab research provides the opportunity to develop and refine such techniques. 
Finally, the question of reliable, agnostic biomarkers is of high interest. Among the proposed biosignatures are large isotope fractionations, unusual isotopic distributions, organic molecular chemical complexity/morphology/compartmentalization, and enantiomeric excess (e.g., Neveu et al., 2018). Therefore, possible alteration by planetary environmental conditions of proposed biosignatures must be evaluated in laboratories to confirm their use. An example of such alteration is the changes in chirality induced by ionizing radiation. Isovaline, an amino acid, has been studied at low temperatures, but few others have (Hudson et al., 2009). Exposure of isovaline molecules of either $\mathrm{R}$ or $\mathrm{S}$ chirality to ionizing radiation near $100 \mathrm{~K}$ converts them into a 50:50 mixture of the two types. In other words, a 50:50 R:S mixture of amino acids on an ocean-world surface exposed to radiation does not preclude homochirality, as in terrestrial biology, in a liquid-phase sub-surface region. The reaction rates for such changes need to be quantified, but seldom have been. The laboratory measurements are straightforward, but time-consuming and require multiple skills and techniques. Continued laboratory research and tandem technology development can streamline analytical techniques to study proposed biosignatures.

\section{Some Uncertainties}

Several concerns need addressing beyond those already mentioned. One is that there can be a discontinuity between the low-temperature icy surfaces of some ocean worlds and conditions needed for in situ chemical analyses, which often must rely on the liquid or gas phase. The possibility that uncertainties can be introduced by the liquid- and gas-phase methods of analyzing solids is by no means new, but one that must be studied with each new method proposed. A well-known example is the uncertainty introduced into some of the Viking astrobiology experiments in which solidphase materials were examined by the introduction of liquid water.

Another concern is the possible discontinuity between sub-surface chemistry and surface observations. This is particularly important for ocean worlds as the rate of upwelling and resurfacing from below are poorly known. Conversely, the movement of surface material that has been altered (e.g., surface radiolysis) to sub-surface depths cannot be ignored. As an example, $\mathrm{SO}_{4}{ }^{2-}$ (sulfate) is formed by the action of magnetospheric radiation on Europa's surface and could serve as an oxidant if transported to sub-surface regions, providing a nutrient for sulfate-reducing bacteria (Loeffler and Hudson, 2015). Therefore, understanding the creation, cycling, and fate of $\mathrm{SO}_{4}{ }^{2-}$ is important for evaluating Europa's habitability. As another example, crystalline solids such as hydrates and clathrates are subject to photolytic and radiolytic amorphization, which destroys their ability to retain gases, yet little is known of the extent or rate of such changes. Laboratory experiments can evaluate such processes over a range of chemical, pressure, temperature, and radiation conditions, as well as over potential anaerobic and aerobic biological metabolisms. 


\section{Recommendations for Ocean-Worlds Laboratory Studies}

Based on the above, we make these recommendations for advancing laboratory research in support of ocean-worlds exploration:

1) Support laboratory research into ocean-worlds environments and processes. The paucity of data from ocean-worlds targets, combined with short mission durations and limited communication windows, necessitates a broad understanding of the chemistry, physics, geology, and potentially the biology possible in these unique environments in order to set attainable science goals for NASA missions.

2) Support the development and use of mission-relevant instruments and techniques in laboratory research, which will assist further development of flightready techniques and technology. Such work often falls in the gaps between pure research and technology development, but it is critical for preparing the scientific community for discoveries in new environments

3) Establish greater cooperation among laboratories specializing in different analytical methods and different capabilities. Ocean-worlds environments possess many unknowns, requiring comprehensive and collective measurements to understand fully the spectral and other evidence of surface, sub-surface, and above surface processes.

4) Establish and provide long-term support for an open-source spectral library database, including radio- and microwave spectroscopy, IR spectroscopy, Raman spectroscopy, and mass spectrometry to assist in data sharing.

References: Barnett, I. L., Lignell, A., Gudipati, M. S., Astrophys. J., 2012, 747, 1 - Carlson, R. W., Calvin, W. M., Dalton, J. B., Hansen, G. B., Hudson, R. L., et al., "Europa's Surface Composition", Europa, University of Arizona Press, 2009, pp. 283-327 - Castro-Wallace, S. L., Chiu, C. Y., John, K. K. et al., Scientific Reports, 2017, 17, 18022 - Gerakines, P. A., Hudson, R. L., Moore, M. H., Icarus, 2012, 220, 647 - Hudson, R. L., Moore, M. H., Gerakines, P. A., JGR-Planets, 2001, 106, 33275 - Hudson, R. L., Lewis, A. S., Moore, M. H., Dworkin, J. P., Martin, M. P., "Enigmatic Isovaline: Investigating the Stability, Racemization, and Formation of a Non-Biological Meteoritic Amino Acid", Bioastronomy 2007: Molecules, Microbes, and Extraterrestrial Life, ASP, 2009, 420, 157 - Johnson, S. S., Anslyn, E. V., Graham, H. V., et al., Astrobiology, 2018, 18, 915 - Klenner, F., et al., Astrobiology, 2020, 20, 1 - Loeffler, M., Hudson, R. L., Icarus, 2013, 224, 257 - Loeffler, M., Hudson, R. L., Astrobiology, 2015, 15, 453 - Moore, M. H., Hudson R. L., Icarus, 2000, 145, 282 - Neveu, M., Hays, L. E., Voytek, M. A., et al., Astrobiology, 2018, 18, 1375 - Thelen, A. E., Nixon, C. A., Chanover, N. J., Cordiner, M. A., et al., Icarus, 2019, 319, 417 - Trainer, M. G., Jimenez, J. L., Yung, Y. L., et al., Astrobiology, 2012, 12, 315

$(7 / 14 / 2020)$ 